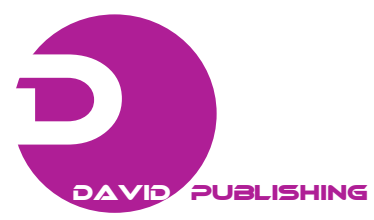

\title{
Optimizing Thermal Energy Storage Systems in the Hospitality Industry
}

\author{
Patrick Wilkinson, Jesse Oshiro, Dean Perry and Helen Lieng \\ California Energy Services Group, The Trane Company, Los Angeles 91748, USA
}

Received: August 02, 2014 / Accepted: September 30, 2014 / Published: December 31, 2014.

\begin{abstract}
Building energy analyses using forecasting optimization strategies are commonly used for predicting TES (thermal energy storage) system performance. These strategies produce perfect optimized cost savings and are not typically realized in the real world, unless a safety factor is applied. Rather than show how to improve the industry's ability to accurately model and simulate a true TES system design, this paper will show advanced building information strategies and energy management simulation techniques required to truly achieve the ideal optimized cost savings, determined from the TES energy simulation analysis. This paper uses the hospitality industry as a case study, showing the application of simulation and analytical modeling for an optimized partial TES system. As a result, building energy managers can make better decisions through the entire building life cycle from the earliest concept model through operation and maintenance.
\end{abstract}

Key words: Thermal storage, energy, controls, monitoring, optimization, 15-min interval data.

\section{Introduction}

Simulation tools are commonly used for predicting TES (thermal energy storage) system performance. These tools are used to determine load profiles, tank charging strategies and most importantly, discharge strategies. Due to the advanced forecasting optimization strategies available in certain simulation software, partial TES systems are able to show optimized savings by forecasting how much storage capacity is required to meet the accumulated chilled water load during a particular discharge period. If the storage capacity available in a particular discharge period is less than that required to meet all of the chilled water load for that period, the program will determine what constant value of the chilled water load can be met by mechanical cooling each hour of the discharge period, so that the thermal storage tank will have discharged to zero by the end of the last discharge

Corresponding author: Patrick Wilkinson, district energy services engineering manager, research fields: energy optimization modeling, and HVAC design. E-mail: patrick.wilkinson@trane.com. period. The major issue with this optimized analysis is that in the real world, it is very complicated to predict the building load profile for each discharge period, and therefore, difficult to optimize when to load chillers and at what tonnage, etc.. There is a disconnection between building design, operational decisions and the predicted simulation modeling results which leads to unsatisfied owners who do not achieve the promised cost savings.

Using the hospitality industry as a case study, this paper uses advanced building information strategies and energy management simulation techniques to realize the ideal optimized cost savings determined from the TES energy simulation analysis. These strategies and techniques include using interval energy optics and real time weather data to provide quick, non-invasive energy evaluations of the building, focusing on energy use patterns and behaviors. Other strategies include using cloud-based building energy management services that help operators make more informed and cost effective decisions about managing and maintaining the building. Intelligent service, 
another strategy, combines technology, analytics, and expertise to continuously monitor, collect, and interpret building data and actively deliver alarm notifications, along with analyzed data and performance-driven recommendations and actions. Then, the building energy manager can make better decisions throughout the entire building life cycle while the owners achieve realized energy savings. Adding TES to an HVAC (heating ventilation air conditioning) system can reduce energy costs associated with comfort cooling by shifting equipment operation from high- to low-cost times of day [1]. Typically, this practice is only economically feasible where the local utility company offers incentives in the form of rate structures or rebates. Many utility companies face capacity shortages during a relatively short period each day-typically, during normal working hours. To encourage users to reduce their energy consumption, the local utility often imposes time-of-day rates that are highest during peak demand periods. One way to curtail the energy costs incurred at these higher rates is to operate the cooling equipment when utility rates are low (usually during the evening) and store the thermal energy potential until it is needed.

Ice tanks are often used as a means of thermal storage because of their compact size and pre-engineered design. During off-peak periods (when utility rates are low), the chiller cools a glycol-water mixture to a temperature of $23{ }^{\circ} \mathrm{F}$. Then, this mixture circulates through tubes within the ice tank, where it freezes the surrounding water. When cooling is needed during an on-peak period (when utility rates are high), the glycol-water mixture, heated by the building load, circulates through the storage tanks to melt the ice.

The tanks required for TES incur additional capital/construction costs. However, such systems often require less chiller capacity, resulting in a smaller chiller selection, i.e., reduced equipment cost. The initial capital cost may be reduced further through the availability of negotiable rebates from the local utility.

\section{Example Utility Rate Structure}

Table 1 describes a typical demand rate structure for a cooling-dominate summer peak region that is ideal for a TES project.

\section{Discharge Strategies}

There are various ways to control and discharge a TES system. The following strategies are commonly implemented and widely simulated in various energy modeling software programs.

\subsection{Optimize}

The program will forecast how much storage capacity is required to meet the accumulated chilled water load during a particular discharge period. If the storage capacity required for a particular discharge period is greater than that required to meet the entire chilled water load for that period, only storage energy is used to meet the chilled water load during those hours. On the other hand, if the storage capacity required in a particular discharge period is less than that required to meet the entire chilled water load for that period, the program will determine what constant value of the chilled water load can be met by mechanical cooling each hour of the discharge period, such that the thermal storage tank will have discharged to zero by the end of the last discharge period.

Table 1 Cost of electricity demand.

\begin{tabular}{|c|c|}
\hline Typical time-of-use schedule - general serv & - large \\
\hline \multicolumn{2}{|l|}{ June through September } \\
\hline Service charge (per month) & $\$ 336.45$ \\
\hline \multicolumn{2}{|l|}{ Demand charge (per kW) } \\
\hline Per $\mathrm{kW}$ for all $\mathrm{kW}$ of billing demand & $\$ 13.73$ \\
\hline Per $\mathrm{kW}$ for all $\mathrm{kW}$ of on-peak billing demand & $\$ 20.94$ \\
\hline Per $\mathrm{kW}$ for all $\mathrm{kW}$ of mid-peak billing demand & $\$ 5.87$ \\
\hline \multicolumn{2}{|l|}{ October through May } \\
\hline Service charge (per month) & $\$ 336.45$ \\
\hline \multicolumn{2}{|l|}{ Demand charge (per kW) } \\
\hline Per $\mathrm{kW}$ for all $\mathrm{kW}$ of billing demand & $\$ 13.73$ \\
\hline Per $\mathrm{kW}$ for all $\mathrm{kW}$ of mid-peak billing demand & $\$ 0.00$ \\
\hline Per $\mathrm{kW}$ for all $\mathrm{kW}$ of off-peak billing demand & $\$ 0.00$ \\
\hline
\end{tabular}




\subsection{Storage Priority}

The storage tank will try to meet the entire chilled water load each hour. If the chilled water load exceeds the available storage capacity, the storage tank is discharged to zero capacity and mechanical cooling is brought online to satisfy the load.

\subsection{Equipment Priority}

Mechanical cooling will meet the chilled water load up to a specific base load defined by the discharge limit. When the chilled water load for a particular hour exceeds the base load, the storage tank is brought online to meet the excess load.

If operated correctly, the optimized discharge strategy will yield the most energy cost savings as the tank's capacity, if required, is completely discharged at the end of the discharge period. If required, the chillers are base loaded to the lowest constant load, therefore, they will minimize overall demand of the central plant.

\subsection{Related Work}

(1) Thermal Storage Engineered Systems Clinics (Trane literature \# ISS-CLC-1, ISS-CLC-2, ISS-CLC-3, and ISS-CLC-4);

(2) Control of Ice Storage Systems Applications Engineering Manual (Trane literature \# ICS-AM-4);

(3) Ice Storage Systems Applications Engineering Manual (Trane literature \# SYS-AM-10).

\section{Building Information Strategies \& Energy Management Services}

\subsection{Energy Optics}

Energy optics leverages existing smart meter interval data to provide a quick, non-invasive evaluation of a building's energy performance through pattern recognition. It provides graphical representations of demand profiles (see Fig. 1) that can quickly compare a building's performance under similar operating conditions, such as degree days, days of the week, and time of the year (see Fig. 2).
Energy professionals utilize the various representations to analyze energy usage patterns and behavior in order to benchmark baseline performance, identify optimal building performance under similar operating conditions, and determine opportunities for improvement, which can then be quantified as energy savings.

\subsection{Cloud-Based Building Energy Management Services}

A cloud-based energy management system gives building owners and operators the ability to make smarter, more cost effective decisions about managing and maintaining buildings. Remote access to building and user-specific energy dashboards, key performance indicator reports, data trending, fault detection and analytics are all provided through a secure web portal (see Fig. 3).

Energy dashboards can be tailored to meet specific needs. Owner dashboards can be created to monitor energy usage and costs either at a building or portfolio level. Facility/energy manager dashboards can be used to display real-time energy consumption, aggregated from utility meters or sub-meters, along with system equipment operating parameters to continuously optimize and reduce energy consumption. Occupant dashboards can be used to monitor energy usage and carbon emissions.

Endless commissioning is made possible through real-time monitoring of equipment operation, equipment specific fault detection and analytics, alarm notification to reveal operational anomalies and associated costs/savings (see Fig. 4). Energy reporting and data trending can be used to track the effectiveness of energy efficiency projects, retrofits or renewable [2].

\subsection{Intelligent Services}

Intelligent services combines technology, proprietary analytics, and industry expertise to continuously collect, interpret, and act on building data, enabling enhanced building performance. Through continuous automated system monitoring and analytics, energy 
professionals can develop value-based actionable recommendations. Continuous commissioning of system parameters provides an assurance that buildings maintain optimal performance, reducing possibilities of operational drift.

A proper intelligent services program is designed to control energy costs, maintain a healthy work environment, keep tenants comfortable, avoid building downtime and reduce operational costs. By proactively identifying operational anomalies, an intelligent services platform successfully reduces energy use and maintains efficient system performance, which enhances the building environment and positively impacts the building occupants.

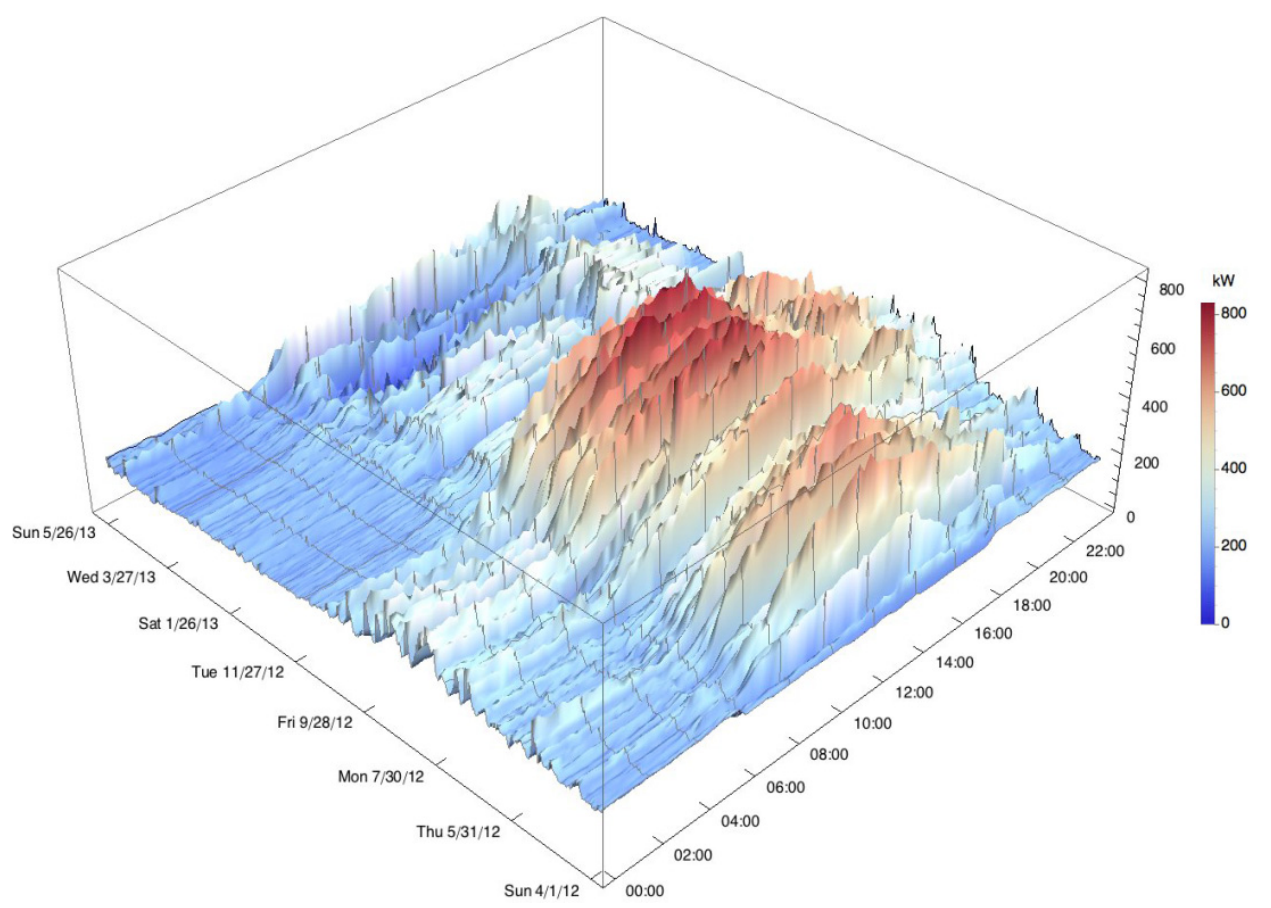

Fig. 1 Energy optics 15-min interval analysis 3D demand profile.

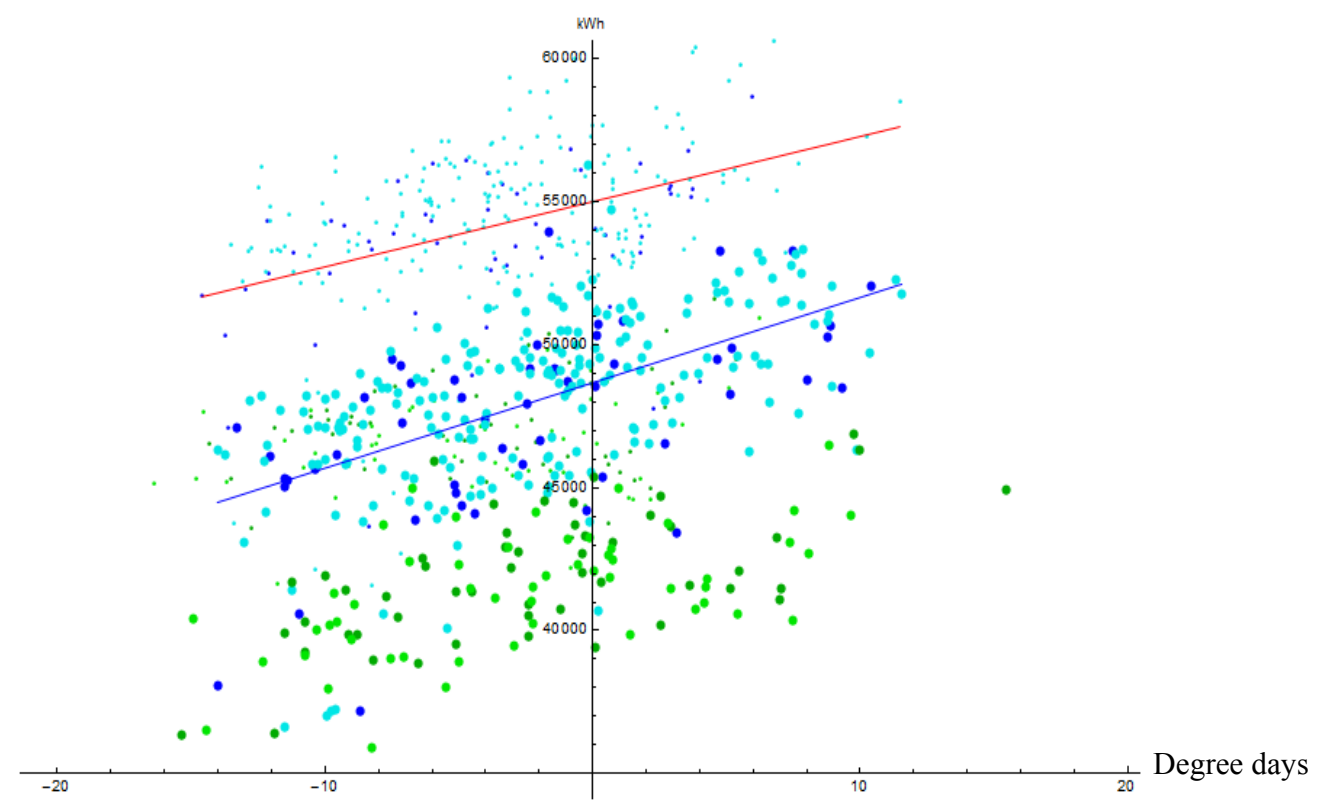

Fig. 2 Energy optics 15-min interval analysis $\mathrm{kWh}$ vs. degree days (color represents type of day). 


\section{Hospitality Case Study}

A single building, multi-purpose facility implemented a partial TES strategy in order to significantly shift demand from the on-peak to off-peak period. A $500 \mathrm{t}$ centrifugal chiller, extremely efficient both in light and full load conditions and equipped with a VFD (variable frequency drive), was installed. A second, $285 \mathrm{t}$ chiller was installed for ice-making operation. The ice making chiller operates at night to charge the 16 TES tanks, which then discharge during the on peak period (noon to $6 \mathrm{pm}$ ) to provide cooling in parallel with the $500 \mathrm{t}$

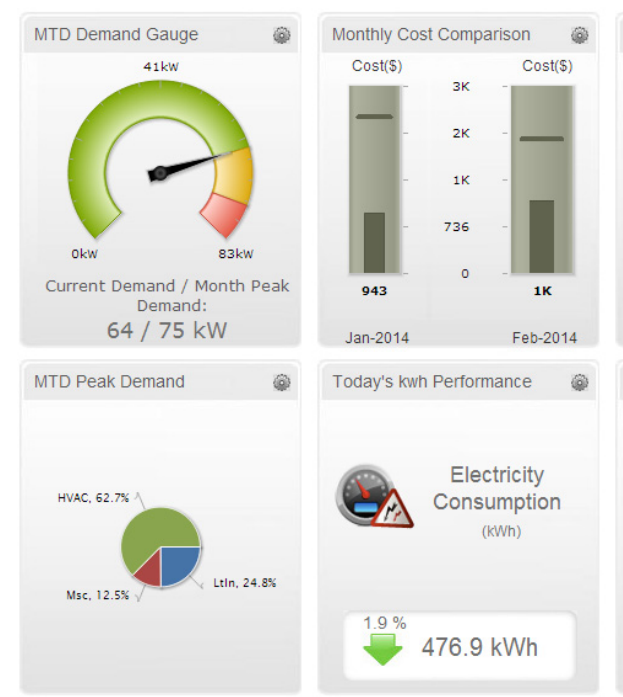

chiller. The required capacity and demand of the $500 \mathrm{t}$ chiller are subsequently reduced. An existing third 350 $\mathrm{t}$ chiller remains strictly as a standby unit (see Fig. 5).

\subsection{Hospitality Energy Optics}

During the design/pre-construction phase, the performance of the cooling plant was measured using permanent data loggers and metering devices in order to determine the operating kilowatts of the chillers, pumps, cooling tower fans, air handler fan motors, light fixtures, etc.. These measurements established both the operating baseline of the equipment and the demand

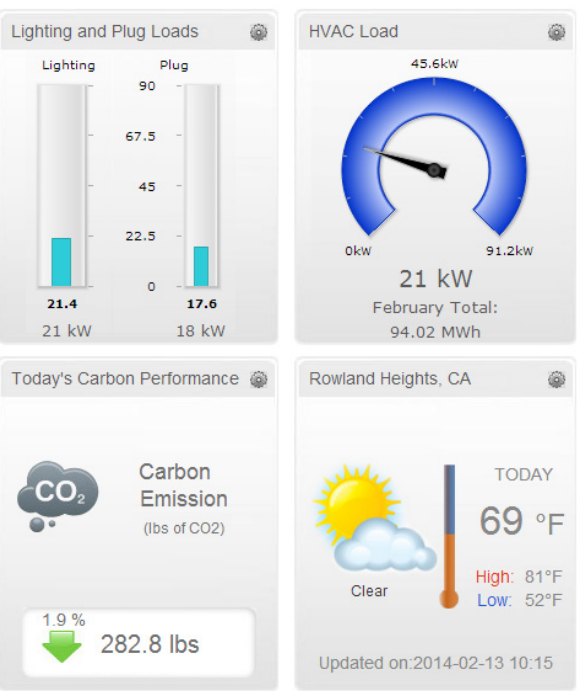

Fig. 3 Energy manager interface.

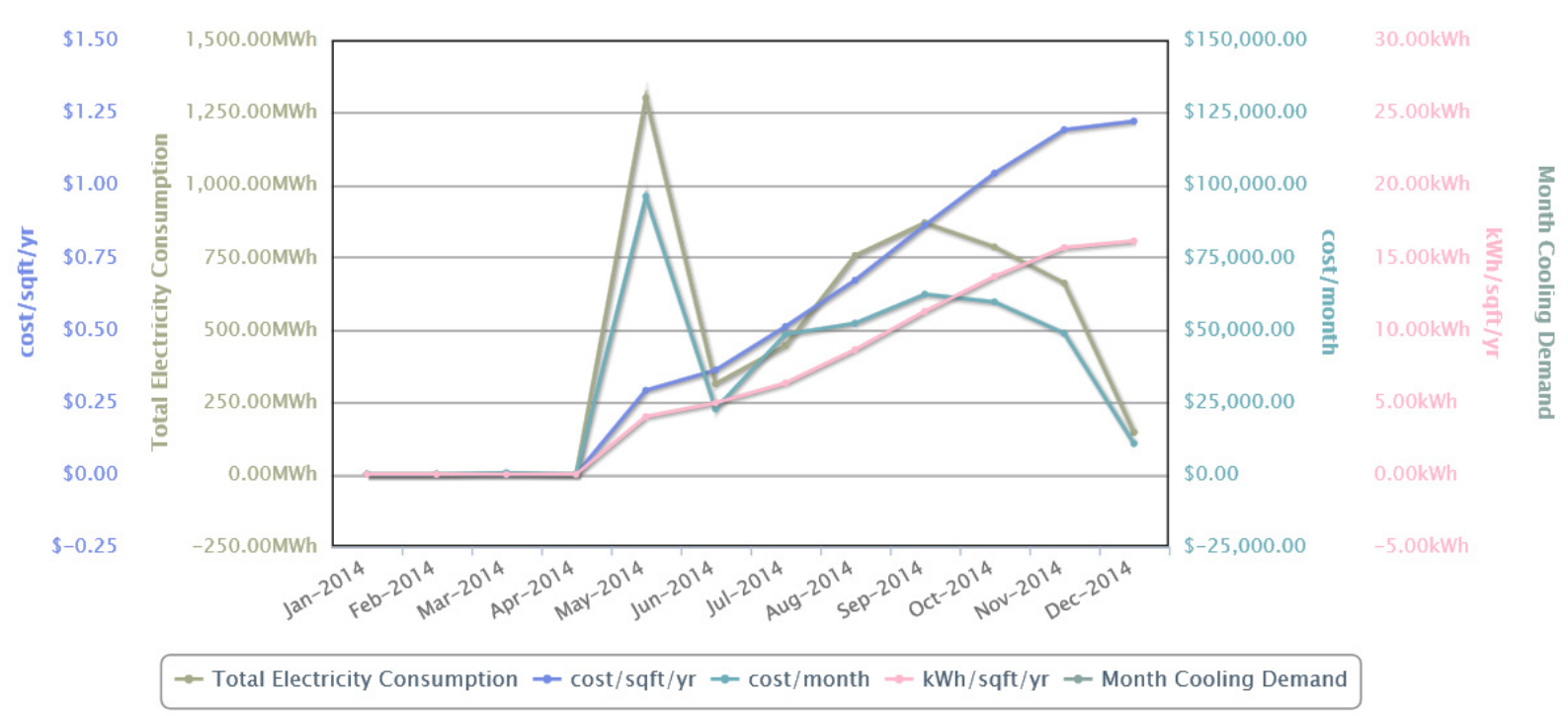

Fig. 4 Energy manager diagnostics. 
profile in energy optics. These measurements will also help pinpoint future deficiencies in the operating characteristics of the new equipment, which will be used to apply corrective action, preventing savings degradation and drift. Details on the measurements are:

- monitoring of the entire central plant's electrical energy consumption;

- separating flow and temperature sensors on the chilled water loops supplying the hotel tower, hotel lobby \& ballrooms area;

- boiler gas consumption monitoring;

- TES flow and temperature sensors on the chilled glycol water loop to determine tank capacity;

- continuous monitoring of all equipment and operations in the facility after the TES installation.

Energy optics allows for a review of the post-construction demand profile to determine any changes that affected the projected energy savings from the original baseline

\subsection{Hospitality Cloud-Based Building Energy} Management Services

Using an energy manager dashboard, the TES operation can be optimized to properly load the chiller

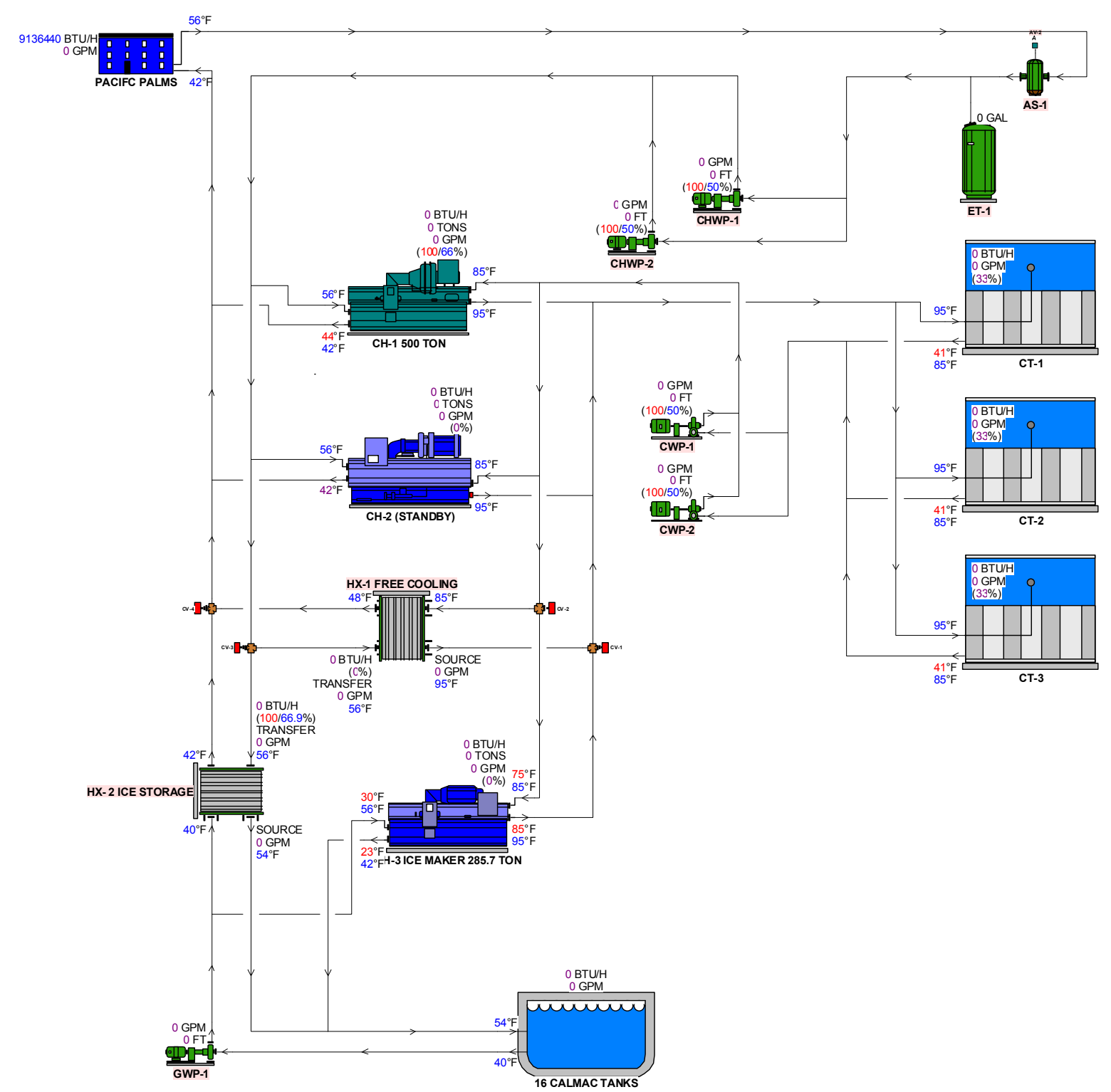

Fig. 5 Central pant schematic. 
while depleting the ice tanks. The listed parameters are used to forecast the accumulated chilled water load during a particular discharge period and how much storage capacity is available to completely deplete the tanks at the end of the discharge period:

- building total cooling load (hotel rooms, ballrooms, lobby);

- real-time ballroom bookings and public space usage;

- occupancy based on real-time reservation levels for hotel rooms;

- current/past weather conditions including daily temperature and humidity profiles;

- previous day cooling load ton-hour profile (12 pm-6 pm).

The following parameters are used to make real time decisions during the discharge period:

- plant and hotel demand (kW);

- plant operation: chiller and ice tank dynamic loading;

- remaining ice storage tank capacity.

The dashboards illustrate the typical layout of the widgets used to optimize the central plant TES system. The dashboard in Fig. 6 is used by the facilities energy manager or a contracted third-party energy manger (hospitality intelligent services). Fig. 7 illustrates the second dashboard is customized by the upper

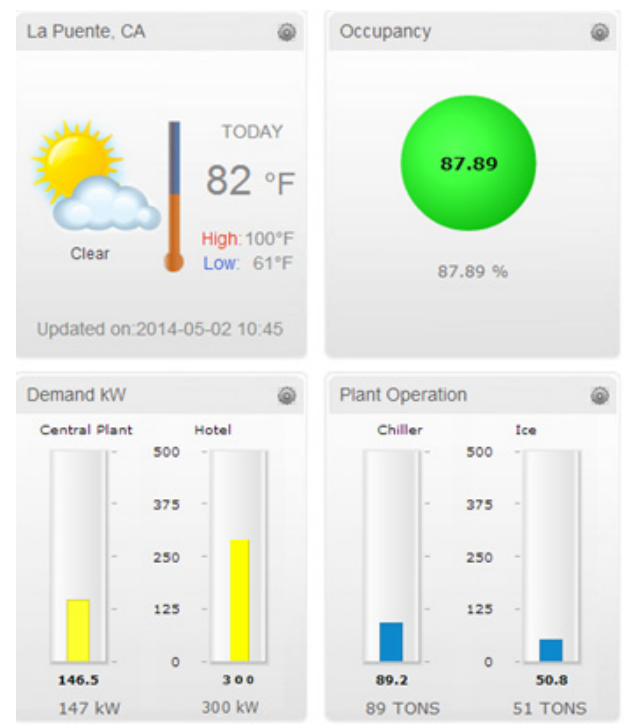

management for key performance indicators needed in the hospitality industry.

\section{Discussion \& Results}

Using the advanced building information strategies and energy management simulation techniques described in this paper, the ideal optimized cost savings determined from the TES energy simulation analysis could be obtained. To maximize savings, parameters and variables are used in the energy manager logic to determine where to load the partial TES system while continuously correcting based on regression analysis (building cooling load as a function of degree days), and trial and error from the results of a sensitivity analysis. The diagrams in Figs. 8-10 illustrate three common depletion scenarios, and the associated cost savings of each outcome can be referenced in Section 7 and Table 2 of this paper.

\section{Conclusion \& Future Work}

Energy savings are determined by comparing the energy use before and after installation of energy conservation measures. In general, this can be represented by Eq. (1):

$$
\text { savings }=(\text { baseline energy use }) \text { adjusted }-
$$$$
\text { (post installation energy use) }
$$

The energy savings are first determined in units of

Fig. 6 Typical energy manager dashboard to control TES optimization.

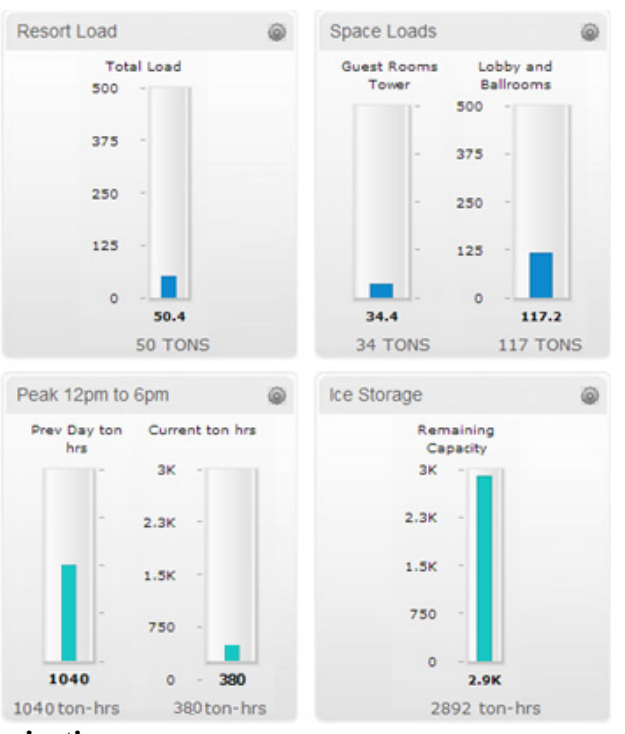



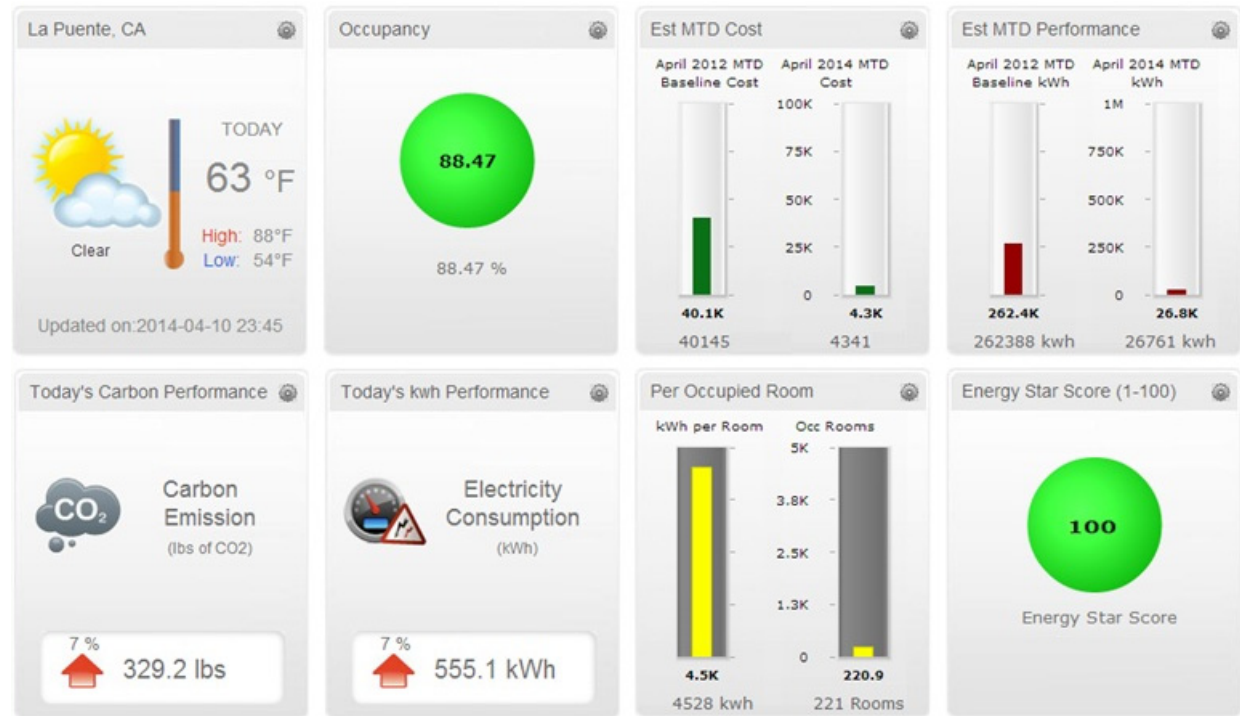

Fig. 7 Typical energy manager dashboard for executive level management.

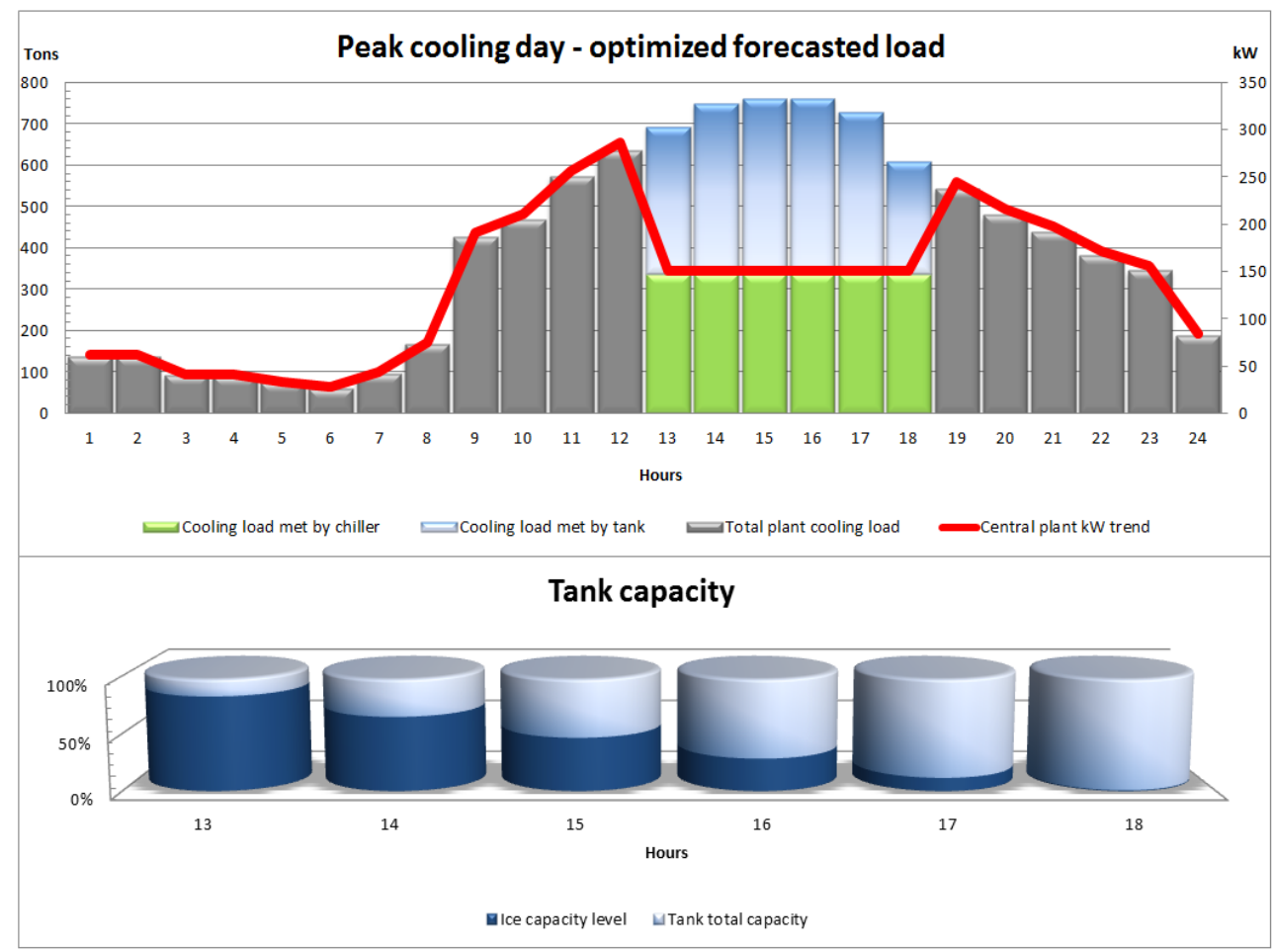

Fig. 8 Cooling load profile with optimized forecasted load.

energy $(\mathrm{kWh})$, energy demand $(\mathrm{kW})$, and therms of natural gas. From these, the dollar amount of energy savings is determined from the utility rate structure. The calculations used in this paper to determine energy savings are based on the IPMVP (International Performance Measurement and Verification Protocol) [3]. The IPMVP is a group of guidelines endorsed by the DOE (Department of Energy) and the ASHRAE (American Society of Heating Refrigeration and Air Conditioning Engineers). The guidelines describe current best practice techniques for verifying the results of energy efficiency and renewable energy projects.

Although the electrical consumption increases 


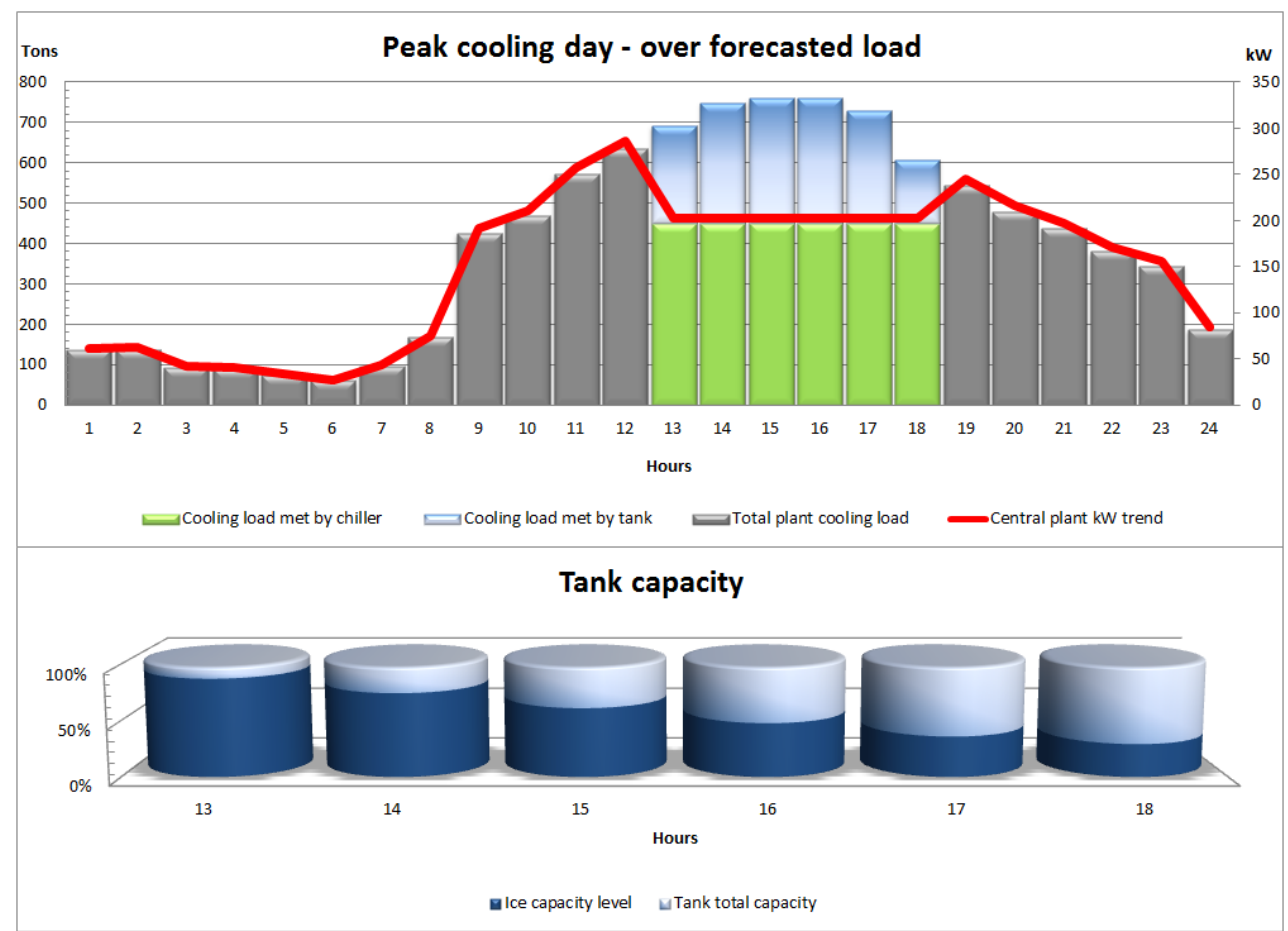

Fig. 9 Cooling load profile with over forecasted load.

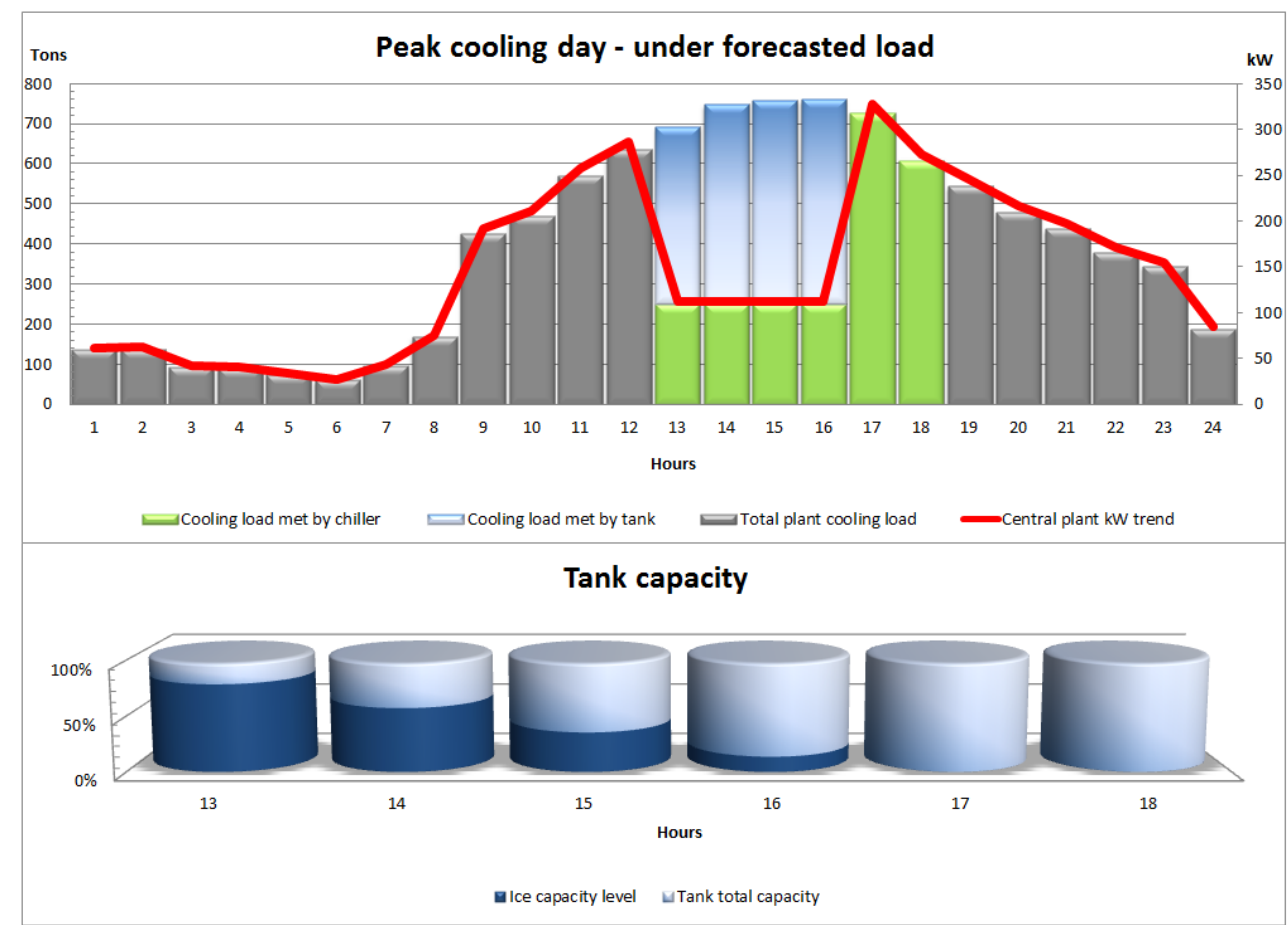

Fig. 10 Cooling load profile with under forecasted load.

Table 2 Annual energy and cost savings from baseline.

\begin{tabular}{llll}
\hline Measure description & Peak demand savings (kW) & Elec. savings (kWh) & Total cost savings \\
\hline Optimized forecasted load & 349.6 & $-105,599$ & $\$ 65,504$ \\
Averaged over forecasted load (250 t base-loaded) & 325.1 & $-94,310$ & $\$ 49,067$ \\
Average under forecasted load (150 t base-loaded) & 57.3 & $-184,297$ & $\$ 20,836$ \\
\hline
\end{tabular}


slightly with the optimized system, it is clear that an optimized system will provide the greatest overall cost savings. The use of advanced building information strategies and energy management simulation techniques is critical for a TES system to provide the most cost savings to building owners.

Future work includes publishing the regression analysis for the independent and dependent variables from the energy manager and how they affect the TES system optimization strategy.

\section{References}

[1] ASHRAE. 2011. ASHRAE Handbook-HVAC Applications. Atlanta: American Society of Heating Refrigeration and Air Conditioning Engineers, Inc..

[2] ASHRAE. 2010. Performance Measurement Protocols for Commercial Buildings. Atlanta: American Society of Heating Refrigeration and Air Conditioning Engineers, Inc..

[3] IPMVP. 2012. Concepts and Options for Determining Energy and Water Savings, Volume I. Toronto: EVO (Efficiency Valuation Organization). 